\title{
Effects of initial crystallinity and molecular orientation on hydrolysis and mechanical properties of self-reinforced poly(lactic acid) screws
}

\author{
Masato SAKAGUCHI* and Satoshi KOBAYASHI* \\ *Department of Mechanical Engineering, Tokyo Metropolitan University \\ 1-1 Minami-Osawa, Hachioji, Tokyo 192-0397, Japan \\ E-mail: sakaguchi-masato@ed.tmu.ac.jp
}

Received 6 November 2015

\begin{abstract}
Poly(lactic acid) (PLA) attracts much attention as a typical biodegradable and biocompatible polymer, because it degrades to nontoxic lactic acid through non-enzymatic hydrolytic degradation. Processing of PLA bone fixation devices have been investigated to improve their mechanical properties. Among fixations, screws are referred as one of the most general bone fixation device. The purpose of the present study is to clarify the effect of initial higher-order structure on degradation behavior and associated mechanical properties for self-reinforced PLA screws. PLA screws were prepared through a series of routes including casting extrusion drawing and forging. These screws were immersed in phosphate buffer solution (PBS) for $0,8,16$ and 24 weeks, and orientation function, crystallinity, molecular weight and shear strength were measured. As a result, initial shear strength increased and degradation ratio decreased with orientation. This is due to lower water absorption caused by orientation crystallization. Shear strength decreased approximately $15 \%$ at least with 24 weeks immersion. The results of the crystallinity showed that the screws were selectively hydrolyzed in amorphous region. This fact suggested that shear strength retention depends on hydrolysis in amorphous region. Initial shear strength for extrusion ratio (ER) 2 is the highest although the orientation function is the lowest. And the strength rapidly decreased with 8 weeks immersion. This might be due to hydrolysis of oriented tie chains. Although $\beta$ crystals emerged only at ER 4, there was not clear difference in molecular weights and shear strength. This result suggested that the crystal form has little effects on degradation behavior and mechanical properties.
\end{abstract}

Key words : Bone fixation device, Poly(lactic acid), Orientation, Mechanical property, Hydrolysis, In vitro

\section{Introduction}

The importance of bone fixation treatment is increasing with development of super aged society. Senior citizens have higher risk of bone fracture because of decrease in body function and/or an osteoporosis. The fracture of thighbone which is necessary for walking is a cause of bedridden of a senior and reduces the quality of life. Traditional metallic bone fixation devices have been used for internal fixation of bone fracture (Champy et al., 1978, Müller et al., 1990, Sugiyama et al., 1999). However, harmful actions such as local osteoporosis, elution of metal ion and metallic allergy would occur when the metallic material presents in the body for a long time (Hofmann, 1992, Rosenberg, 1993, Tomita and Kutsuna, 1987, Uhthoff and Finnegan, 1983). Therefore, the metallic bone fixation devices should be removed after complete healing of bone fracture. This secondary surgery is a burden for patients physically, mentally and economically. Bioabsorbable poly(lactic acid) (PLA) attracts much attention, because of compensation for these demerits of metals.

Biological reaction during PLA degradation seldom occurs and lactic acid which is a product of the decomposition of PLA is nontoxic. Hence, PLA has been clinically used in orthopedic and/or oral-maxillofacial surgeries (Bessho et al., 1997, Böstman et al., 1990, Partio et al., 1992a, 1992b Suuronen, 1993, Yamamuro et al., 1994). However, 
mechanical properties of PLA have not fulfilled the requirements of an application to a higher loaded part (Manninen et al., 1992, Shetty et al., 1997, Wittenberg et al., 1991). Therefore, self-reinforced (SR) PLA has been investigated to improve mechanical properties. Gogolewski et al. (Gogolewski and Pennings, 1983) obtained fibers with a tensile strength up to $1.2 \mathrm{GPa}$ and Young's modulus $15 \mathrm{GPa}$ by hot drawing of fibers spun from solution of PLA. This study evidenced that strength of drawn PLA fibers is dependent on the molecular weight and on the drawing ratio. Hyon et al. (Hyon et al., 2005) investigated mechanical properties of extruded PLA rod through hydrostatic extruding. As a result, the extruded rod achieved more than $350 \mathrm{MPa}$ bending strength which is larger than that of the human cortical bone (200 MPa). The hydrolysis behaviors of SR-PLA bone fixation device has been evaluated experimentally (Hyon et al., 2005, Majola et al., 1992, Pohjonen et al., 1997, Shikinami and Okuno, 1999). Majola et al. (Majola et al., 1992) evaluated the strengths and strength retention of SR-PLA rods after intramedullary and subcutaneous implantation in rabbits. There were no changes in the shear strength during 12 week's hydrolysis. At 36 weeks the bending strength had decreased to the level of the strength of cancellous bone. On the other hand, the effect of higher order structure, especially molecular orientation, on mechanical properties of PLA device during hydrolysis has not been clarified.

Among bone fixations, we focused on bone fixation screws, since screws are referred as one of the most general bone fixation device. The purpose of this study is to clarify the effect of initial higher-order structure on degradation behavior and associated mechanical properties for SR-PLA screw. PLA was extruded and molded to screw. These screws are immersed in simulated body environment and orientation function, crystallinity and shear strength were investigated.

\section{Experiments}

\subsection{Preparation of Poly(lactic acid) Screws}

Commercial PLA pellets (Ingeo 3001D, Nature Works LLC, the USA) were used to manufacture screws. The PLA pellets have weight average molecular weight of about 155,000 , glass-transition temperature of about $73{ }^{\circ} \mathrm{C}$ and melting point of $170{ }^{\circ} \mathrm{C}$. Before molding, PLA pellets were dried in an electric oven at $70{ }^{\circ} \mathrm{C}$ for more than 7 hours. Cylindrical billets were molded of the pellets by casting. The PLA pellets were molten in the single-screw extruding machine (PPKR-mini, Imoto Machinery) and were casted in a mold of billet. After the molten pellet was filled in the mold, the mold were cold pressed under room temperature. Molding conditions of casting are molding temperature of $200{ }^{\circ} \mathrm{C}$, rotation number of screw of $100 \mathrm{rpm}$, pressure of cold press of $40 \mathrm{MPa}$, respectively.

The billets were drawn by extrusion drawing. Figure 1 gives a schematic representation of a mold used for extrusion drawing. Lubricant, polyethylene glycol, was sprayed on the taper part of the mold heated to extrusion temperature. Then, PLA billet was set in the taper part and heated for 10 minutes. Compressive loading was applied to the heated billet by using a push part with a hot press system. The extruded rod with the mold was taken from the hot press system and was cooled on an aluminum plate until $60{ }^{\circ} \mathrm{C}$ or below for about 7 minutes. Extrusion condition is extrusion ratio (ER) of 1, 1.3, 2, 4 and 8 and extrusion temperature of $105^{\circ} \mathrm{C}$. ER for extruded rods was determined as the ratio of cross sectional area of cylindrical billet to that of extruded rod, whose ratio corresponds to the ratio of the length of an extruded rod to the original length. These extruded rods had the length of $40 \mathrm{~mm}$ and diameter of $6 \mathrm{~mm}$ for all ER. To obtain the rods of same dimension for all ER, dimensions of billet for each ER were determined as shown in Table 1.

Threads were molded on the surface of a rod by forging with a mold as shown in Fig. 2. The rod was set in a set of heated mold which has thread, and heated for 10 minutes. Threads on the mold were transcribed on the heated rod by cold pressing. Forging conditions are temperature of $120{ }^{\circ} \mathrm{C}$ and compression load of $200 \mathrm{kN}$. At last, burrs on the screw were removed by using a die. The size of a screw was M6 and thread length of a screw was $30 \mathrm{~mm}$.

Table 1 Dimensions of billet for each ER which molded by extrusion molding.

\begin{tabular}{c|c|c|c|c|c}
\hline Extrusion Ratio & 1 & 1.3 & 2 & 4 & 8 \\
\hline Diameter $[\mathrm{mm}]$ & 6 & 7 & 8.5 & 12 & 17 \\
\hline Length $[\mathrm{mm}]$ & 40 & 30 & 21 & 11.5 & 6.5 \\
\hline
\end{tabular}




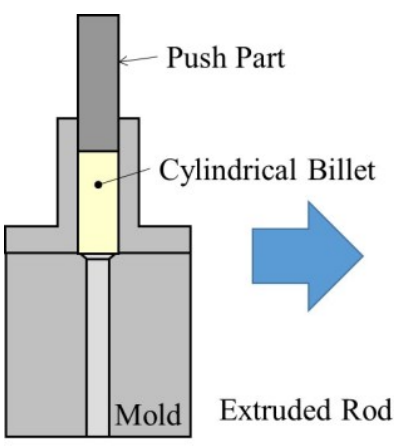

(a) Before Drawing

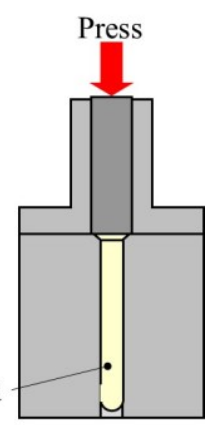

(b) After Drawing

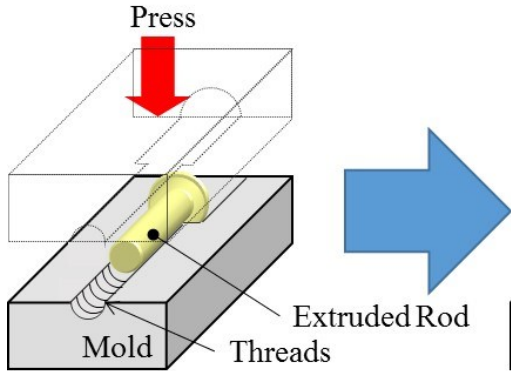

(a) Before Forging

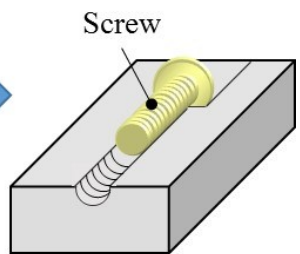

(b) After Forging

Fig. 1 Sectional view of mold used in the extrusion drawing.

\subsection{In vitro Degradation of PLA Screws}

In vitro degradation tests were conducted in phosphate buffered solution (PBS) at pH 7.4 and $37{ }^{\circ} \mathrm{C}$. PBS was prepared with phosphate buffer powder (Wako Pure Chemical Industries) to concentration of 1/15 mol/1 in pure water. PLA screws were immersed for 0, 8, 16 and 24 weeks. PBS was changed weekly to avoid the effect of acidic product of decomposition from the screws.

Weight of PLA screws was measured to determine water absorption ratio. The weight was determined with an accuracy of $0.1 \mathrm{mg}$ after wiping PBS on the screw. The measurement was carried out daily until the weight became stable and weekly after that. The water absorption ratio was calculated following to,

$$
c=\frac{m_{2}-m_{1}}{m_{1}} \times 100
$$

where $c$ is water absorption ratio [\%], $m_{1}$ and $m_{2}$ are weights of screw before and after immersion [g], respectively.

\subsection{Measurement of Molecular Weights}

Molecular weights of immersed screws were determined by gel permeation chromatography (GPC). A specimen was cut to approximately $0.4 \mathrm{~g}$, and dried in vacuum at room temperature for more than 12 hours. Then the specimen was completely dissolved in chloroform of approximately $20 \mathrm{ml}$ and the solution was filtered through a filter with pore size of $0.45 \mu \mathrm{m}$ (PTFE Membrane, Whatman). Weight and number average molecular weight and polydispersity in the solution were evaluated with a Shimadzu GPC system with columns (Shim-pack GPC804C and GPC801C, Shimadzu).

\subsection{Measurement of Orientation Function}

Chain orientation in screw axis was characterized with a measurement of an orientation function using polarized infrared (IR) spectroscopy. The orientation function is defined from angle between polymer chain axis and drawing direction. The value of orientation function 1 and 0 corresponds to perfect alignment and random orientations, respectively. IR spectra were recorded using a Fourier transform infrared spectrometer (IR Prestige-21, Shimadzu) and single reflection type attenuated total reflection measuring device (MIRacle A, PIKE Technology). The specimen was buried in epoxy matrix, then cut in the screw axis direction as a shown Fig. 3. The cross section was polished by using emery papers and was buffed to mirror surface with aluminum powder. IR spectra were measured using polarized infrared beam in the parallel and perpendicular direction to specimen axis as shown in Fig. 3. An oriented specimen consisting of $\alpha$ crystals exhibits the absorption peak at $923 \mathrm{~cm}^{-1}$ that is assigned to the $\mathrm{CH}_{3}$ rocking mode showing transition moment perpendicular to chain axis (Kang et al., 2001). IR dichroic ratio $R$ is determined by dividing an absorbance of polarized infrared parallel by that of infrared polarization perpendicular for screw axis direction. Crystalline chain orientation function $f_{\mathrm{c}}$ was calculated from $R$ by using follow equation,

$$
f_{c}=\frac{R-1}{R+2} \frac{R_{0}+2}{R_{0}-1}
$$


where $R_{0}$ is $2 \cot ^{2} \phi$. The $\phi$ is angle between transition moment associated with considered absorption and molecular axis, where $\phi$ is $90^{\circ}$ (Sawai et al., 2003).

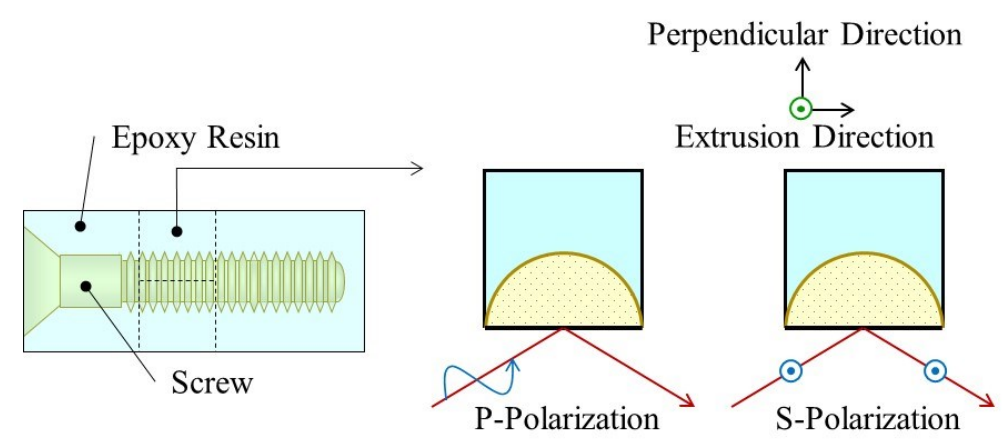

Fig. 3 Schematic view of specimen which was embedded in epoxy resin and polarized direction of infrared light.

\subsection{Measurement of Crystallinity}

Crystallinity of a screw was determined from thermograms measured with a differential scanning calorimeter (DSC-60, Shimadzu). The specimen was dried in vacuum at room temperature for more than 12 hours and was cut to approximately $0.4 \mathrm{mg}$. The DSC measurement was carried out from room temperature to $230{ }^{\circ} \mathrm{C}$ at heating rate of 10 ${ }^{\circ} \mathrm{C} / \mathrm{min}$, using air as a reference material. The fusion and the crystallization enthalpy $\left(\Delta H_{m}\right.$ and $\Delta H_{c}$, respectively) were calculated from the fusion and crystalline peaks of this curve. The crystallinity $X_{\mathrm{c}}$ was calculated using the following equation,

$$
X_{c}=\frac{\Delta H_{m}+\Delta H_{c}}{135} \times 100
$$

where the value $135 \mathrm{~J} / \mathrm{g}$ is the theoretical enthalpy of completely crystalline PLA (Miyata and Masuko, 1998).

\subsection{Shear Testing Method}

Shear strength of a PLA screw was measured with an apparatus designed in our laboratory. Figure 4 shows a schematic view of the apparatus where a specimen is set. The apparatus consisted of convex and concave parts. A screw hole was introduced in each part where the specimen was inserted. The apparatus was subjected to compressive loading during the test using a universal testing machine (AGS-X, Shimadzu) operated at a cross head speed of $0.5 \mathrm{~mm} / \mathrm{min}$. Thereby, shear loading was applied in the cross-sections perpendicular to the axis of the screw between concave and convex parts as shown in Fig. 4. Shear strength of screw was calculated as maximum force divided by an effective area. The effective area is $45 \mathrm{~mm}^{2}$ since the size of screw is M6 and shear loading is held in two testing areas.

Acoustic emission (AE) which generated during a shear test was measured to investigate fracture behavior of the screw. An AE sensor (AE-900M Type I, NF Corporation) was bonded as shown in Fig. 4. Frequency response of the $\mathrm{AE}$ sensor ranged from $300 \mathrm{kHz}$ to $2000 \mathrm{kHz}$. Discrimination high and low levels as thresholds were $30 \mathrm{mV}$ and 20 $\mathrm{mV}$, respectively. Total gain of amplifier was set to $60 \mathrm{~dB}$. AE measurement system which was used in this study outputs the number, the energy and the maximum amplitude of AE signal at constant interval. The interval was set to $100 \mathrm{~ms}$. 


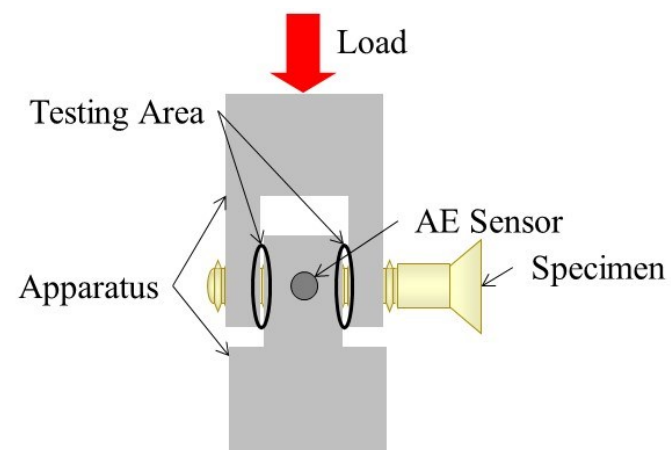

Fig. 4 Schematic view of shear test and the position of AE sensor.

\section{Experimental Results}

\subsection{Observation and Water Absorption Ratio}

Appearances of screws before and after immersion are shown in Fig. 5. The color of screws after immersion became whiter than that before immersion. The whitening was observed from 8 weeks after immersion, and do not change due to immersion time. The whitening might be due to increase crystallinity during immersion. Figure 6 shows water absorption ratio of screws which was calculated from result of weight measurement during immersion. The absorption ratio increased quickly for first 1 week in each ER, and increased gradually until 24 weeks. Initial rapid increase in absorption ratio was due to diffusion of water to molecular chains. Successive gradual increase after the rapid increase depended on loosening of molecular entanglement due to plasticizing effect which offer the site of water adsorption. In comparison with ER, the absorption ratios at ER 1 is higher than that of ER 1.3 for overall immersion time. This is due to difficulty of water absorption by orientation crystallization. Molecules of water could not diffuse in crystal region, because the molecular chains of specimen are densely packed (Li et al., 1990). Therefore, water absorption ratio decreased by drawing.

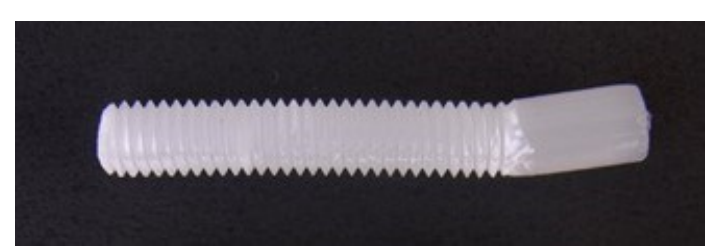

(1) Extrusion Ratio 1,0 week

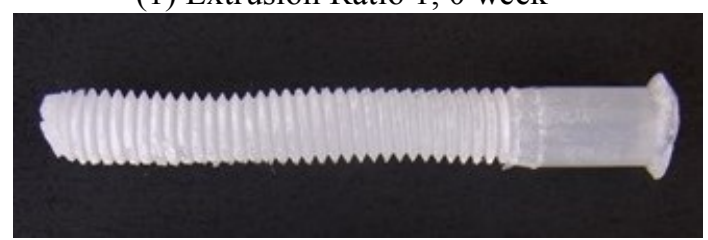

(2) Extrusion Ratio 2, 0 week

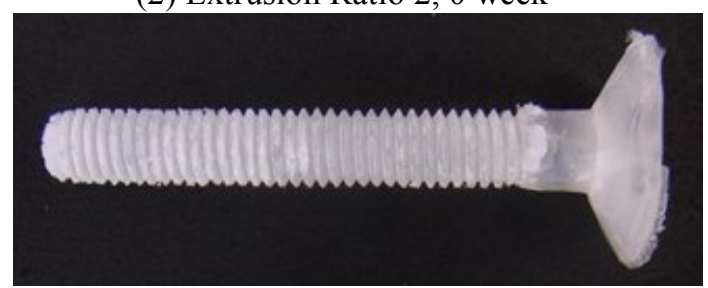

(3) Extrusion Ratio 8, 0 week

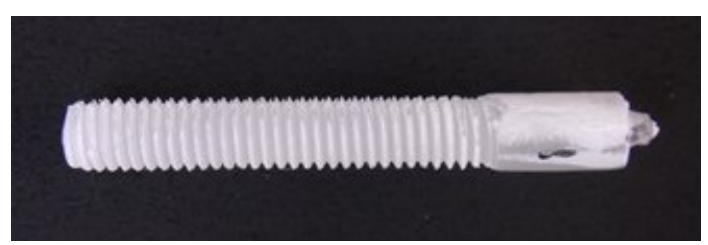

(4) Extrusion Ratio 1, 24 week

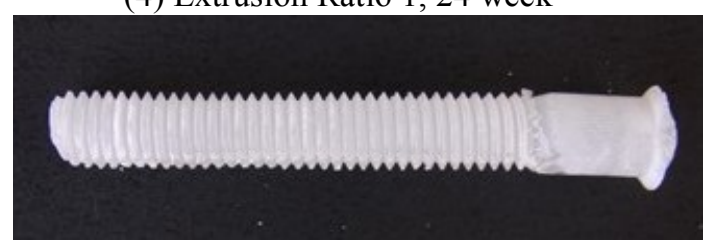

(5) Extrusion Ratio 2, 24 week

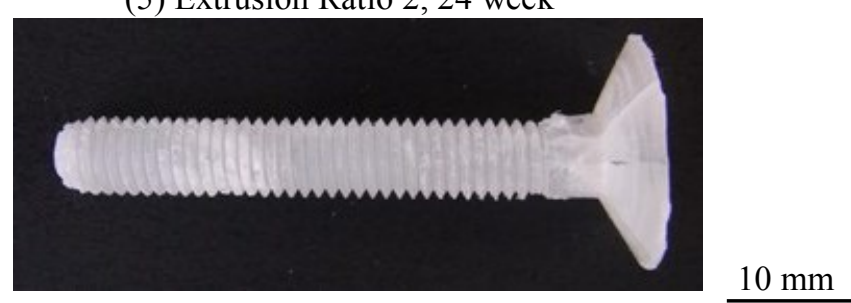

(6) Extrusion Ratio 8, 24 week

Fig. 5 Screw before and after immersion in phosphate buffered solution. 


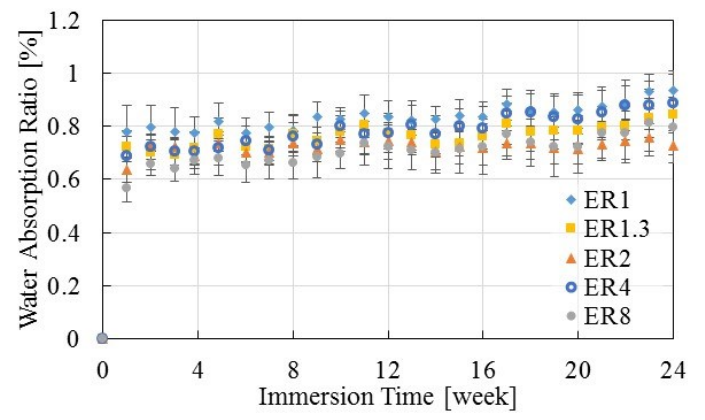

Fig. 6 Water absorption ratio of screws with extrusion ratio (ER) 1 to 8 as a function of immersion time.

\subsection{Molecular Weight}

Weight and number average molecular weight and polydispersity for screws which immersed for 0 to 24 weeks are shown in Fig. 7 to 9, respectively. Both of the weight and the number average molecular weight decreased with increasing immersion time. This suggests that screws degraded by hydrolysis. For each ER, there was not significant difference in the both molecular weights at 0 week, but molecular weights for higher ER were higher than that for lower ER with increasing immersion time. This is due to be lower water absorption caused by orientation crystallization, and suggests that degradation rate was lower with increasing ER. The crystal region remains during hydrolysis of PLA, because of selective hydrolysis of amorphous region. The peak in distribution of molecular weight from the remained crystal region occurs at low molecular weight side of the distribution (Tsuji et al., 2000). Therefore, polydispersity increases by hydrolysis. However, there were no change in the polydispersity with immersion time. This is because 24 weeks immersion time was too short to degrade molecular in crystallites.

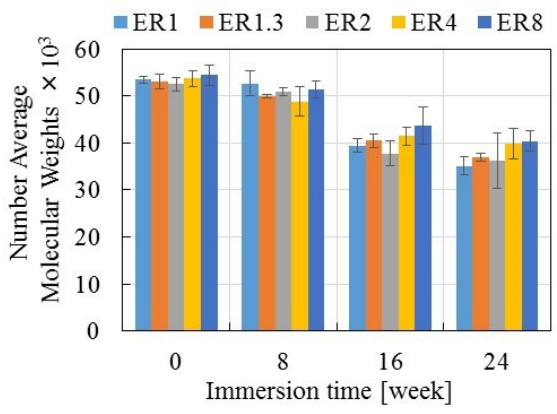

Fig. 7 Number average molecular weight

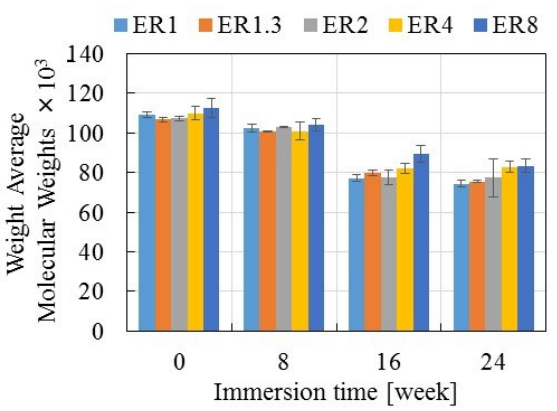

Fig. 8 Weight average molecular weight.

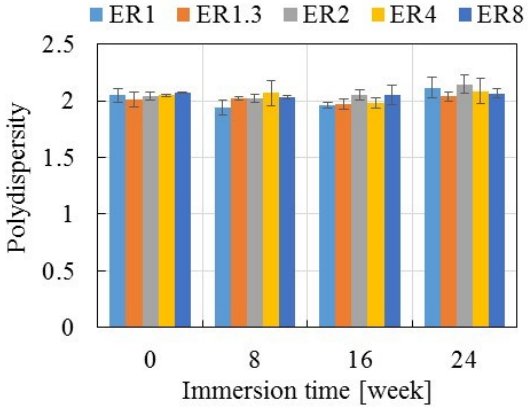

Fig. 9 Polydispersity.

\subsection{Orientation Function}

Figure 10 shows polarized IR spectra which were obtained for screws before immersion. The IR spectra show that the peak height at $923 \mathrm{~cm}^{-1}$ was different to polarized direction on extruded screw. This corresponded to orientation for $\alpha$ crystal by extrusion drawing. A peak at $912 \mathrm{~cm}^{-1}$ is shown on the extruded screw at ER 4 . The peak is assigned to the $\mathrm{CH}_{3}$ rocking mode of $\beta$ crystals by Sawai et al. (Sawai et al., 2003).

Figure 11 shows orientation functions which were calculated from IR spectra. The orientation function decreased with ER for ER 1 to 2 and increased for ER 2 to 4. This is due to orientation by material flow during casting Orientation functions of casted billets are shown in Fig. 12. The orientation functions of billets decreased with increasing ER. This is because dimension of billets varied with ER. In the present study, the diameter and length of the billet became thinner and longer with decreasing ER to assure a constant dimension of the rod after drawing at different ER. Molecular chains were highly oriented by material flow during casting from an extruder with longer billets. This orientation remains in screws after extrusion drawing and forging. Hence, orientation function at ER 1 is higher than that at ER 1.3 and 2.

The orientation function for ER 8 decreased during immersion. This might be due to crystallization during immersion. The orientation function decreased with increasing non-oriented crystal during this crystallization. On the other hand, the decrease in orientation function for ER 4 was smaller than that for ER 8. This might be attributed to the 
difference in crystal form. The crystallization during immersion proceeded with growth of existing crystal and formation of new crystalline. The result of IR spectra showed formation of $\beta$ form crystalline for ER 4 . The growth of $\beta$ crystal does not affect orientation function of $\alpha$ crystal. Hence, the decrease in orientation function for ER 4 is little.

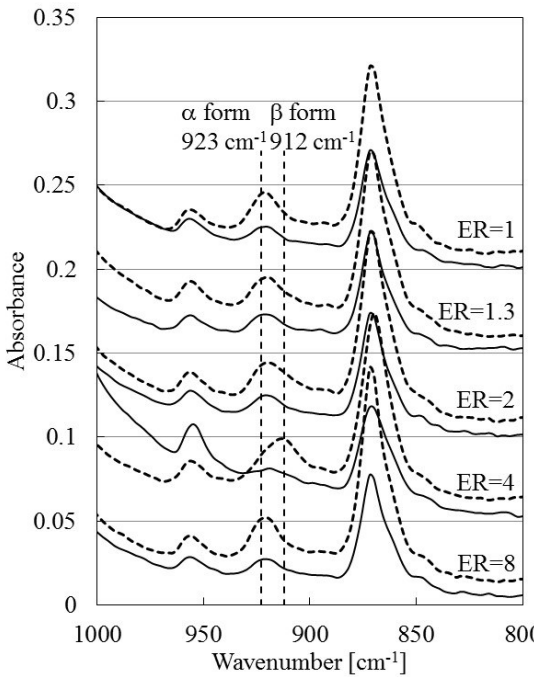

Fig. 10 IR spectra for each ER measured with the incident beam polarized both parallel (-) and perpendicular ( $\cdots)$ to the extrusion direction.

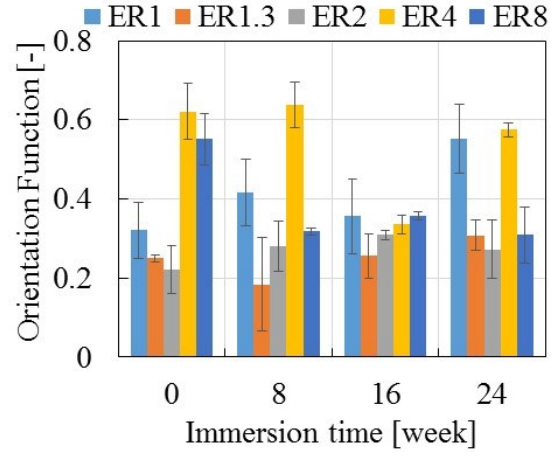

Fig. 11 Orientation functions on immersed screws.

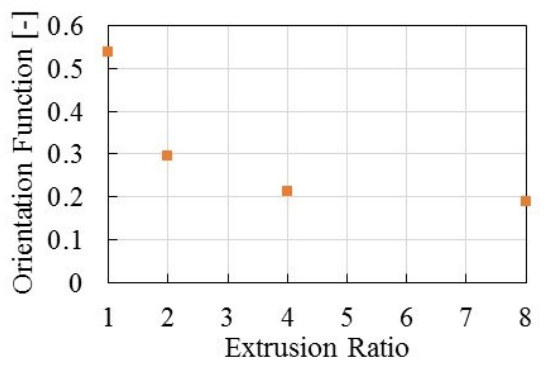

Fig. 12 Orientation functions on billets before drawing.

\subsection{Crystallinity}

The DSC thermograms for screws before immersion are shown in Fig. 13. The screw with any ER did not show a crystallization peak around $110^{\circ} \mathrm{C}$. This indicates that crystallization saturated during extrusion drawing and forging. The screw with ER 4 exhibited a small peak around $156^{\circ} \mathrm{C}$ in addition to a main peak around $168{ }^{\circ} \mathrm{C}$. The two peaks around $156{ }^{\circ} \mathrm{C}$ and $168{ }^{\circ} \mathrm{C}$ are due to the melting of $\alpha$ crystals and $\beta$ crystals, respectively. This result corresponded to IR spectra in Fig. 10 and a previous report (Hyon et al., 2005). However, the screw with ER 8 did not shown a peak due to the melting of $\beta$ crystals. This result suggests that $\alpha$ crystal transition due to heat generation by friction and/or larger deformation was dominant during extrusion to ER 8.

Crystallinities in the immersed screws for 0 to 24 weeks are shown in Fig. 14. The crystallinities in the screws before immersion are approximately $33 \%$ for each ER. With immersion, the crystallinities increased to the range $34 \%$ to $39 \%$. This is due to selective hydrolysis of amorphous region in the screws. However, the crystallinity at ER 8 which has high orientation function is the second highest in 24 weeks. This might be due to increasing crystallinity during immersion. This increasing is due to the increase in mobility of PLA molecular chains caused by water molecules diffused in specimen.

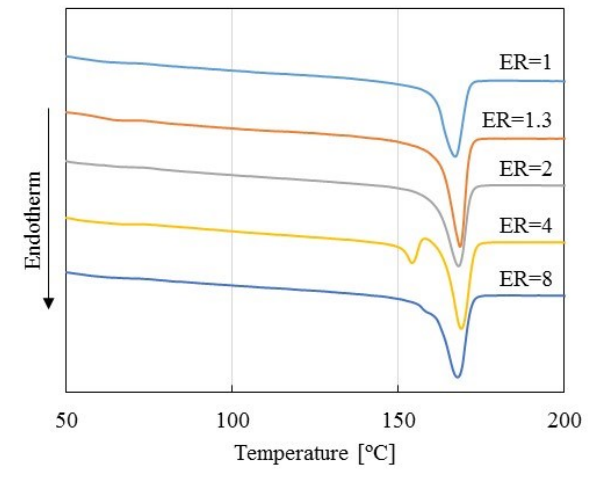

Fig. 13 DSC thermogram for each ER.

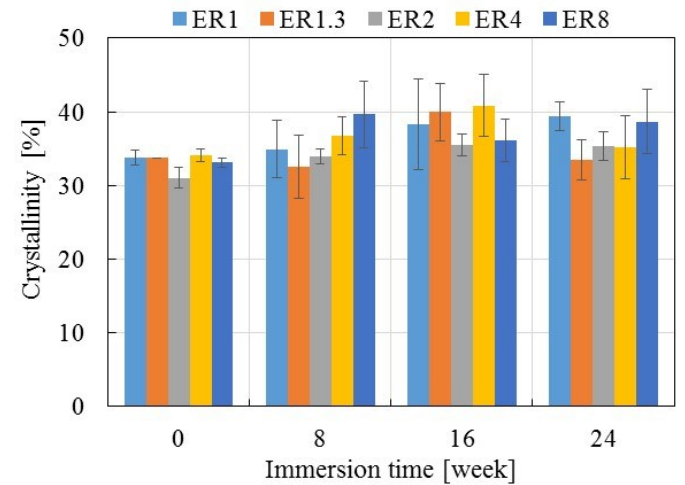

Fig. 14 Crystallinities in immersed screws. 


\subsection{Shear testing}

Figure 15 shows shear stress versus testing time curves which were obtained from immersed screws. Figure 15 consists of typical result for ER 1, 2 and 8. The graphs also include cumulative AE energy and event and maximum amplitude of AE wave. Amplitude shows the maximum amplitude of AE signal at constant interval. And, cumulative AE energy and event curve show cumulative values of the energy and the number of AE signal. The slope for stress-testing time decreased at about $40 \mathrm{MPa}$ in each specimen. Large amplitude generated around the knee point of stress-time curve at ER 1. Cumulative AE energy and event did not increase at ER 1. Cumulative AE event at ER 2 began to increase for approximately $200 \mathrm{~s}$ of testing time. Cumulative AE energy or event at ER 8 began to increase for $100 \mathrm{~s}$ to $200 \mathrm{~s}$ of testing time. In comparison with ER, large amplitude increased with ER. In comparison with immersion time, AE generation behavior did not change with immersion time.

Figure 16 shows shear strength of immersed screws for 0 to 24 weeks with ER 1 to 8 . Initial shear strength slightly decreased with ER 1 to 1.3 and increased for ER 1.3 to 8. Shear strength for each ER decreased with immersion time. Shear strength for more than ER 2 remained approximately $80 \mathrm{MPa}$ for 24 weeks. This value is close to strength for self-reinforced screw investigated by Suuronen et al. (Suuronen et al., 1992). The residual strength for ER 1 after 24 weeks immersion decreased to $71 \%$ of strength before immersion, whereas the residual strengths for ER 4 and 8 is $85 \%$ and $87 \%$, respectively.

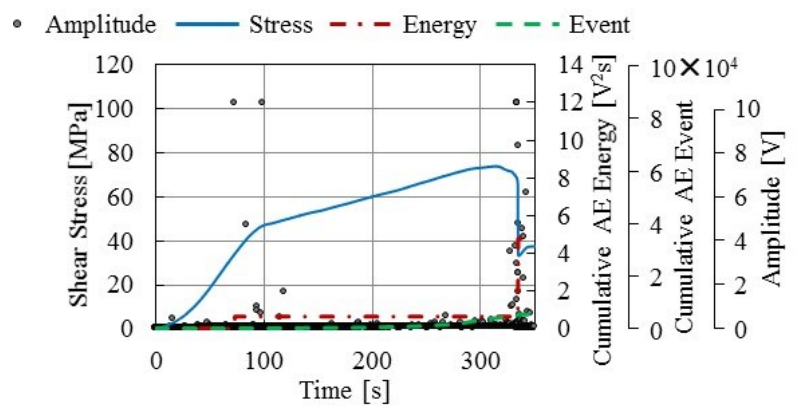

(1) ER1, 8 week

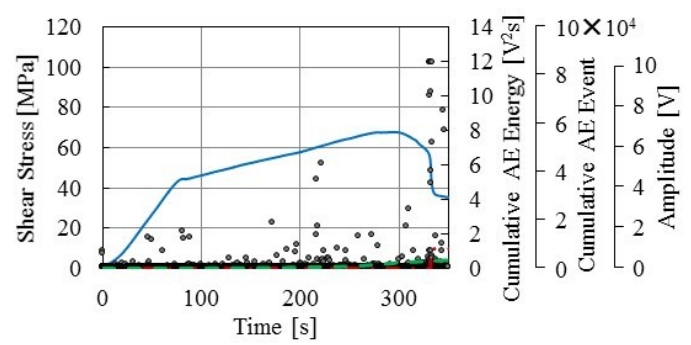

(3) ER1, 24 week

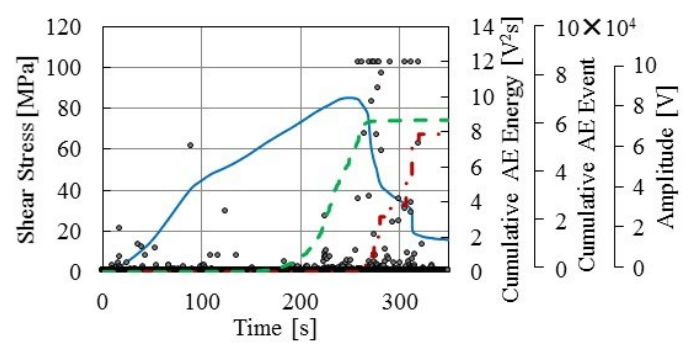

(5) ER2, 16 week

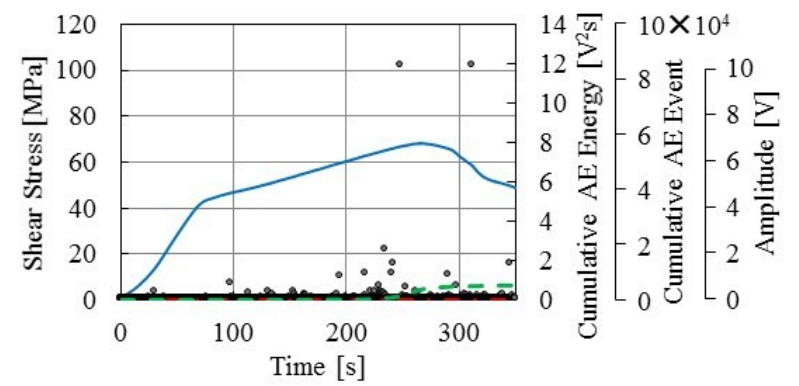

(2) ER1, 16 week

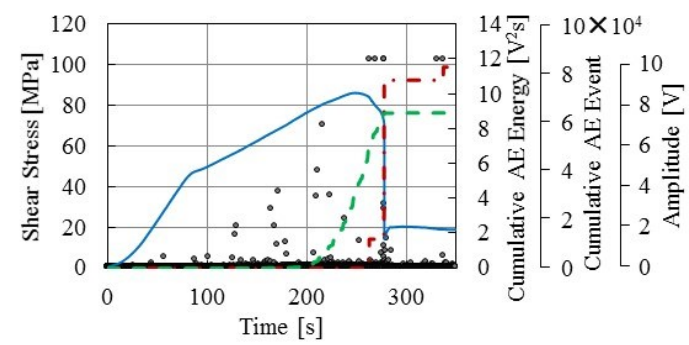

(4) ER2, 8 week

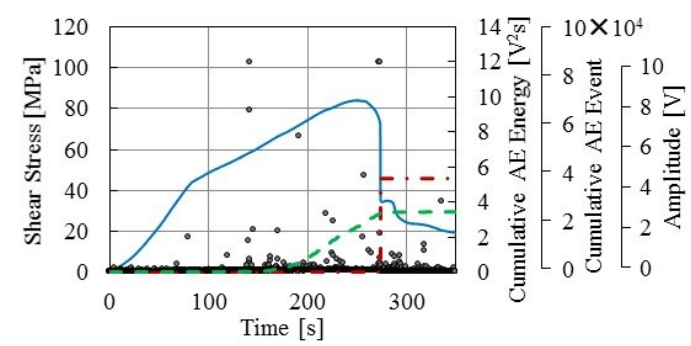

(6) ER2, 24 week 


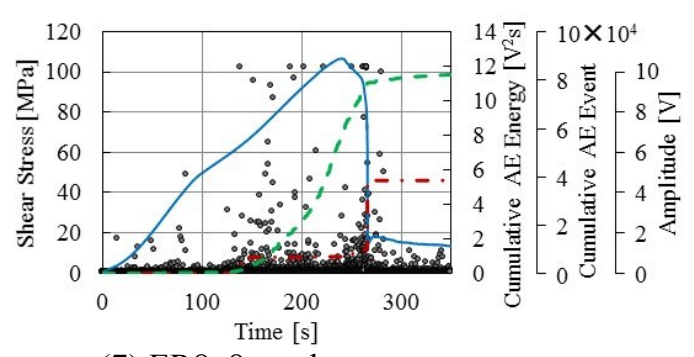

(7) ER8, 8 week

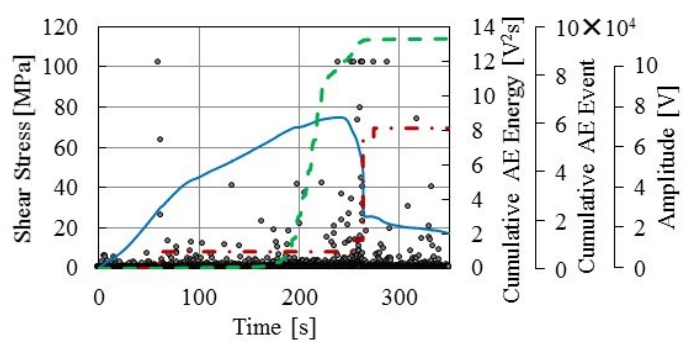

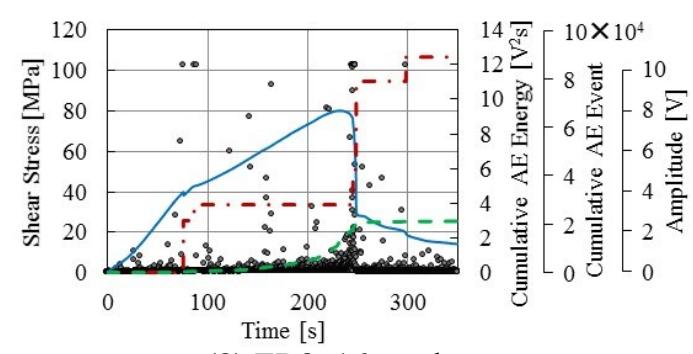

(8) ER8, 16 week

(9) ER8, 24 week

Fig. 15 Relationship between shear stress, cumulative AE energy, event, AE amplitude and testing time on the immersed screw.

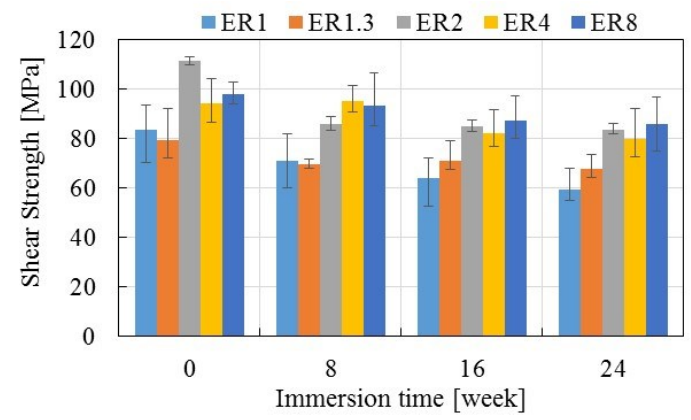

Fig. 16 Shear strength of screws with each ER for each immersion time.

\section{Discussion}

\subsection{Effect of Extrusion Ratio on Fracture Behavior of Self-Reinforced PLA Screw}

AE generation corresponds to occurrance of microscopic damages such as micro-crack and/or craze in screws during shear testing. Therefore, fracture behavior of a screw in shear test was speculated as follows. Microscopic damages occurred by shear loading, which fact resulted in generation of acoustic wave with large amplitude around the knee point of stress-time curve at ER 1 and increasing cumulative AE event at ER 2 and 8. Microscopic damages accumulated and grew until maximum stress, which fact resulted in increasing cumulative AE event. At the point where shear stress rapidly decreases, cumulative AE energy and event rapidly increased. This corresponded to breaking of the screw. The number of larger amplitude acoustic wave increased with ER. The result suggests that microscopic damage during shear testing increases with ER. AE generation behavior did not change with immersion time. The result suggests that fracture behavior of a screw did not change with immersion time.

\subsection{Effect of in vitro Degradation on Mechanical Properties of Self-Reinforced PLA Screws 4.2.1 Effect of molecular orientation on initial shear strength of screw}

Initial shear strength and orientation function of screw showed similar tendency. Increasing shear strength with drawing might be due to formation of fibrous crystals. The fibrous crystals were formed by stretching and rearranging molecular chains during the drawing and had high tensile strength in the orientation direction and high shear strength in the perpendicular direction to orientation. Therefore, the increase in strength is due to molecular orientation during extrusion drawing. 
However, initial shear strength for ER 2 is highest although the orientation function is lowest. The shear strength at ER 2 rapidly decreased with 8 weeks immersion. Hence, high initial shear strength at ER 2 might be caused by oriented tie chains in amorphous region between crystalline which chains was degraded during immersion. Decreasing shear strength after immersion is due to degradation of the tie chain. Shear strengths for ER 4 and 8 are lower than that for ER 2. This is because tie chain was pulled out from crystal during large deformation for ER 4 and/or 8.

\subsubsection{Effect of molecular orientation on immersed shear strength of screw}

Shear strength for each ER decreased with immersion time. Since molecular weight also decreased with immersion time, this is due to hydrolysis during immersion. The residual strength after 24 weeks immersion increased from $71 \%$ for ER 1 to $87 \%$ for ER 8. From the results, it is confirmed that degradation ratio decreases with orientation crystallization. This is due to decreasing water absorption ratio by orientation crystallization during the drawing and suppression of decreasing molecular weight. Hence, degradation rate for shear strength decreased with ER, and the strength for extruded screw remained higher than that for non-extruded screw.

\section{Conclusion}

In order to clarify the effect of initial higher-order structure on degradation behavior and associated mechanical properties for SR-PLA screw, immersion and shear tests were conducted on PLA screws fabricated through drawing and forging processes. Increasing orientation during extrusion drawing increased initial shear strength and prevent the decrease in molecular weight to improve the shear strength retention. This is due to lower water absorption caused by orientation crystallization. This structural transformation during extrusion drawing affected occurrence and development of microscopic damages during shear testing.

Shear strength was sensitive to microscopic structure of amorphous region. Initial shear strength for ER 2 is highest although the orientation function is lowest. The shear strength for ER 2 rapidly decreased with 8 weeks immersion. These results suggested that the highest initial shear strength for ER 2 might be caused by oriented tie chain between crystalline which chain degraded during immersion.

\section{References}

Bessho, K., Iizuka, T. and Murakami, K., A bioabsorbable poly-L-lactide miniplate and screw system for osteosynthesis in oral and maxillofacial surgery, Journal of Oral and Maxillofacial Surgery, Vol. 55(1997), pp. 941-945.

Böstman O., Hirvensalo E., Mäkinen J., Rokkanen P., Foreign-body reactions to fracture fixation implants of biodegradable synthetic polymers, The Journal of Bone and Joint Surgery. British Volume, Vol. 72(1990), pp. 592-596.

Champy, M., Loddé, J. P., Schmitt, R., Jaeger, J. H. and Muster, D., Mandibular osteosynthesis by miniature screwed plates via a buccal approach, Journal of Maxillofacial Surgery, Vol. 6(1978), pp. 14-21.

Gogolewski, S. and Pennings, A. J., Resorbable materials of poly(L-lactide). II. Fibers spun from solutions of poly(L-lactide) in good solvents, Journal of Applied Polymer Science, Vol. 28 (1983), pp. 1045-1061.

Hofmann, G. O., Biodegradable implants in orthopaedic surgery-A review on the state-of-the-art, Clinical Materials, Vol. 10(1992), pp. 75-80.

Hyon, S. H., Jin, F., Moon, S. I. and Tsutsumi, S., Hydrostatic extrusion of poly(L-lactide), Macromolecular Symposia, Vol. 224 (2005), pp. 93-104.

Kang, S., Hsu, S.L., Stidham, H. D., Smith, P. B., Leugers, M. A. and Yang, X., A spectroscopic analysis of poly(lactic acid) structure, Macromolecues, Vol. 34(2001), pp. 4542-4548.

Li S. M., Garreau H., Vert M., Structure-property relationships in the case of the degradation of massive aliphatic poly-( $\alpha$-hydroxy acids) in aqueous media, Journal of Materials Science: Materials in Medicine, Vol. 1(1990), pp. 123-130.

Majola, A., Vainionpää, S., Rokkanen, P., Mikkola, H. M. and Törmälä P., Absorbable self-reinforced polylactide (SR-PLA) composite rods for fracture fixation : strength and strength retention in the bone and subcutaneous 
tissue of rabbits, Journal of Materials Science: Materials in Medicine, Vol. 3(1992), pp. 43-47.

Manninen, M. J., Päivärinta, U., Taurio, R., Törmälä, P., Suuronen, R., Räihä, J., Rokkanen, P. and Pätiälä, H., Polylactide screws in the fixation of olecranon osteotomies, Acta Orthopaedica Scandinavica, Vol. 63(1992), pp. 437-442.

Miyata, T. and Masuko, T., Crystallization behavior of poly(L-lactide), Polymer, Vol. 39(1998), pp. 5515-5521.

Müller, M. E., Allgöwer, M., Schneide, R. and Willenegger, H., Manual of Internal Fixation (3 rd ed) (1990), p. 94, Sringer Verlag.

Partio, E. K., Merikanto, J., Heikkilä, J. T., Ylinen, P., Mäkelä, E. A., Vainio, J., Törmälä, P. and Rokkanen, P., Totally absorbable screws in fixation of subtalar extra articular arthrodesis in children with spastic neuromuscular disease, Journal of Pediatric Orthopaedics, Vol. 12(1992a), pp. 646-650.

Partio, E. K., Hirvensalo, E., Partio, E., Pelttari, S., Partio, K. J., Böstman, O., Hänninen, A., Törmälä, P. and Rokkanen, P., Talocrural arthrodesis with absorbable screws, Acta Orthopaedica Scandinavica, Vol. 63(1992b), pp. 170-172.

Pohjonen, T., Helevirata, P., Törmälä, P., Koskikare, K., Patiala, H. and Rokkanen, P., Strength retention of self-reinforced poly-L-lactide screws, Journal of Materials Science : Materials in Medicine, Vol. 8(1997), pp. 311-320.

Rosenberg, A., Grätz, K. W. and Sailer, H. F., Should titanium miniplates be removed after bone healing is complete?, International Journal of Oral and Maxillofacial Surgery, Vol. 22(1993), pp. 185-188.

Sawai, D., Takahashi, K., Sasashige, A., Kanamoto, T. and Hyon, S. H., Preparation of oriented $\beta$-form poly(L-lactic acid) by solid-state coextrusion: effect of extrusion variables, Macromolecules, Vol. 36(2003), pp. 3601-3605.

Shetty, V., Caputo, A. A. and Kelso, I., Torsion-axial force characteristics of SR-PLLA screws, Journal of Cranio-Maxillofacial Surgery, Vol. 25(1997), pp. 19-23.

Shikinami, Y. and Okuno, M., Bioresorbable devices made of forged composites of hydroxyapatite (HA) particles and poly-L-lactide (PLLA): Part I . Basic characteristics, Biomaterials, Vol. 20(1999), pp. 859-877.

Sugiyama, Y., Ishibashi, S., Sekiyama, S., Sera, K. and Futatsukawa, S., Analysis of elements in the soft tissue covering titanium plates and screws for internal bone fixation by the pixe method, International Journal of PIXE, Vol. 9(1999), pp. 305-313.

Suuronen, R., Pohjonen, T., Taurio, R., Törmälä, P., Wessman, L., Rönkkö, K., Vainionpää, S., Strength retention of self-reinforced poly-L-lactide screws and plates: an in vivo and in vitro study, Journal of Materials Science: Materials in Medicine, Vol. 3(1992), pp. 426-431.

Suuronen, R., Biodegradable fracture-fixation devices in maxillofacial surgery, Journal of Oral and Maxillofacial Surgery, Vol. 22(1993), pp. 50-57.

Tomita, N. and Kutsuna, T., Experimental studies on the use of a cushioned plate for internal fixation, International Orthopaedics, Vol. 11(1987), pp. 135-139.

Tsuji, H. and Ikada, Y., Properties and morphology of poly(l-lactide) 4. Effects of structural parameters on long-term hydrolysis of poly(l-lactide) in phosphate-buffered solution, Polymer Degradation and Stability, Vol. 67(2000), pp. 179-189.

Uhthoff, H. K. and Finnegan, M., The effects of metal plates on post-traumatic remodelling and bone mass, Journal of Bone \& Joint Surgery (British Volume), Vol. 65(1983), pp. 66-71.

Wittenberg, J. M., Wittenberg, R. H. and Hipp, J. A., Biomechanical properties of resorbable poly-L-lactide plate and screws, Journal of Oral and Maxillofacial Surgery, Vol. 49(1991), pp. 512-516.

Yamamuro, T., Matsusue, T., Uchida, A., Shimada, K., Shimozaki, E. and Kitaoka, K., Bioabsorbable osteosynthetic implants of ultra-high strength poly-L-lactide, International Orthopaedics, Vol. 18(1994), pp. 332-40. 